MINI-REVIEW

\section{The role of thrombin and thrombin receptors in ischemic, hemorrhagic and traumatic brain injury: deleterious or protective?}

\author{
Guohua Xi, ${ }^{*}$ Georg Reiser $\dagger$ and Richard F. Keep*† \\ Departments of *Neurosurgery and $\$$ Physiology, University of Michigan, Ann Arbor, Michigan, USA \\ $\dagger$ Institute for Neurobiochemistry, Otto-von-Guericke-University Magdeburg, Magdeburg, Germany
}

injury induced by a large dose of thrombin, an intracerebral hemorrhage or by focal cerebral ischemia. Thrombin may also be an important mediator of ischemic preconditioning. In contrast, high doses of thrombin kill neurons and astrocytes in vitro and cause disruption of the blood-brain barrier, brain edema and seizures in vivo. This review examines the role of thrombin in brain injury and the molecular mechanisms and signaling cascades involved.

Keywords: cerebral hemorrhage, cerebral ischemia, preconditioning, thrombin, thrombin receptors.

J. Neurochem. (2003) 84, 3-9.
There has been much interest in the role that serine proteases play in brain injury. Much of this has focused on tissue plasminogen activator (tPA) because of its use as a therapeutic agent for ischemic stroke (The NINDS rt-PA stroke study group 1995) and because of evidence that suggests that endogenous tPA within the brain may modulate different forms of brain injury (Gingrich and Traynelis 2000). However, there is a growing body of work indicating that another serine protease, thrombin, also plays an important role in modulating brain injury processes and this is the focus of the current review.

Thrombin, a serine protease generated by the cleavage of prothrombin, is an essential component of the coagulation cascade. It is produced in the brain either immediately after a cerebral hemorrhage (either primary or secondary to brain trauma) or after the blood-brain barrier (BBB) breakdown that occurs following many kinds of brain injury (Lee et al. 1996a). Evidence, both in vivo (Nishino et al. 1993; Lee et al. 1996b; Lee et al. 1997a,b; Xi et al. 1998) and in vitro (Vaughan et al. 1995; Striggow et al. 2000; Jiang et al. 2002a), indicates that high concentrations (more than $500 \mathrm{~nm}$ ) of thrombin within the brain parenchyma can be deleterious. Our recent studies have demonstrated that thrombin is responsible for early brain edema formation following intracerebral hemorrhage (ICH) and contributes to ischemic brain injury (Lee et al. 1996b; Striggow et al. 2000; Xi et al. 2002). However, thrombin at very low concentrations (from $50 \mathrm{pm}$ to $100 \mathrm{nM}$ ) is neuroprotective (Vaughan et al. 1995; Xi et al. 1999; Masada et al. 2000; Striggow et al. 2000; Xi et al. 2000). This review examines the role of thrombin in brain injury and the mechanisms and pathways involved.

Received June 4, 2002; revised manuscript received September 17, 2002; accepted October 14, 2002.

Address correspondence and reprint requests to Guohua $\mathrm{Xi}, \mathrm{MD}$, R5550 Kresge I University of Michigan, Ann Arbor, Michigan 481090532, USA. E-mail: guohuaxi@umich.edu

Abbreviations used: AT III, anti-thrombin III; BBB, blood-brain barrier; HIF- $1 \alpha$, hypoxia inducible factor- $1 \alpha$; HO-1, heme oxygenase-1; HSP, heat shock protein; ICH, intracerebral hemorrhage; $\mathrm{IP}_{3}$, inositol 1,4,5-triphosphate; MAPKs, mitogen-activated protein kinases; MMPs, matrix metalloproteinases; PAI-1, plasminogen activator inhibitor-1; PARs, protease-activated receptors; PN-1, protease nexin-1; SAH, subarachnoid hemorrhage; TM, thrombomodulin; tPA, tissue plasminogen activator; TPC, thrombin preconditioning; TRAP, thrombin receptoractivating peptide. 
The dichotomy in the cerebral effects of thrombin may reflect its multiple actions. Thus, thrombin enhances the synthesis and secretion of nerve growth factor in glial cells, modulates neurite outgrowth, reverses process-bearing stellate astrocytes to epithelial-like astrocytes, stimulates astrocyte proliferation and modulates the cytoskeleton of endothelial cells (Grand et al. 1996). It also potentiates NMDA receptor function (Gingrich et al. 2000) and activates rodent microglia in vitro (Moller et al. 2000), factors that may enhance brain injury. Furthermore, a combined intracerebral injection of thrombin and tissue-type plasminogen activator, causes more brain injury than injection of either compound alone (Figueroa et al. 1998). An understanding of these multiple actions of thrombin may lead to potentially novel therapeutic strategies for brain injury, particularly if the thrombin-activated signaling pathways can be elucidated. This might allow selective inhibition of deleterious pathways and activation of protective pathways.

\section{Thrombin in the brain after injury}

Prothrombin, which is synthesized primarily in the liver, is present in plasma at micromolar levels. Activation of either the intrinsic or extrinsic coagulation cascade results in the production of Factor Xa, which cleaves prothrombin to thrombin. Upon clotting, $1 \mathrm{~mL}$ of whole blood can produce about 1-2 nmols of thrombin. Thus, brain injuries that result in the entry of blood into brain, such as a primary intracerebral hemorrhage and brain trauma, result in the immediate production of large amounts of thrombin from cleavage of the prothrombin in the blood. Although it should be noted that the precise perihematomal concentrations have not been measured.

Many ischemic and traumatic brain injuries are associated with BBB disruption without hemorrhage. Evidence suggests that thrombin levels are increased in such tissue (Xi et al. 2002). Because the BBB becomes permeable to large molecular weight proteins in such injuries, this production of thrombin may result from the entry of elements of the coagulation cascade, including prothrombin, from blood. However, the brain may also be a source of prothrombin. In vitro studies have shown that prothrombin mRNA is expressed in the cells of the nervous system (Dihanich et al. 1991). Prothrombin mRNA in the brain is up-regulated after cerebral ischemia and spinal cord injury (Citron et al. 2000; Riek-Burchardt et al. 2002; Xi et al. 2002). These results suggest that thrombin may be formed and cause brain injury even if the BBB is intact, particularly because the mRNA for Factor $X$ is present in brain (Shikamoto and Morita 1999). Whether Factor X can be activated in the brain without the entry of other coagulation factors from blood is unknown. Current information on the presence within brain of elements of the coagulation cascade and their inhibitors is depicted in Fig. 1.

\section{Thrombin receptors in the brain}

Although thrombin has a vital role in hemostasis through cleavage of fibrinogen to fibrin, other important cellular activities of thrombin, such as p44/42 mitogen-activated protein kinases (MAPKs) activation, appear to be receptor mediated. Carney and Cunningham first identified thrombin receptors in 1978 and the first receptor was cloned in 1991 (Vu et al. 1991). Thrombin receptors are seven transmembrane $\mathrm{G}$ protein-coupled receptors that are activated by proteolytic cleavage rather than by ligand binding. Three proteaseactivated receptors (PARs), PAR-1, -3 and -4 , can be activated by thrombin, whereas PAR-2 is activated by trypsin and mast cell tryptase (Coughlin 2000). Thrombin receptor mRNA expression is found in neurons and astrocytes (Weinstein et al. 1995), and there is functional evidence for the presence of thrombin receptors on both cell types (Gingrich and Traynelis 2000; Ubl et al. 2000; Wang et al. 2002).

PAR-1, -3 and -4 are linked to a wide variety of intracellular signaling cascades (Coughlin 2000). Thus, for example, PAR- 1 can couple to members of the $G_{12 / 13}, G_{q}$ and $\mathrm{G}_{\mathrm{i}}$ families, and dependent on which $\mathrm{G}$ protein is coupled it may regulate $\mathrm{Rho}$, inositol 1,4,5-triphosphate $\left(\mathrm{IP}_{3}\right)$ (and

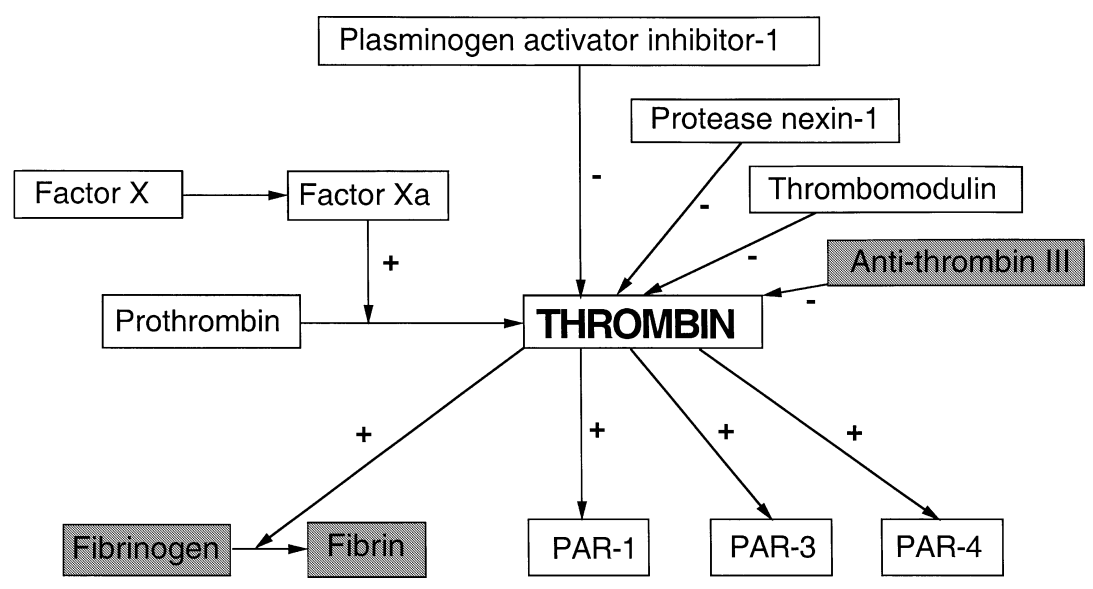

Fig. 1 Elements of thrombin signaling and coagulation cascade, present in the brain with an intact blood-brain barrier (open boxes) and those that may enter brain parenchyma after blood-brain barrier disruption (gray boxes). 
thus $\mathrm{Ca}^{2+}$ ), diacylglycerol, adenylate cyclase and a number of other pathways (Coughlin 2000).

\section{Thrombin inhibitors in the brain}

Understanding the effects of thrombin after brain injury also requires knowledge of endogenous thrombin inhibitors. In the brain, the effects of thrombin are modulated by serine protease inhibitors (serpins) and other thrombin inhibitors such as thrombomodulin. Serpins are a superfamily of proteins including anti-thrombin III (AT III), $\alpha 1$-protease inhibitor, plasminogen activator inhibitor-1 (PAI-1), protease nexin-1 (PN-1), heparin cofactor II and colligin. In the normal brain parenchyma, of the serpins that inhibit thrombin, only PN-1 and PAI-1 appear to be present with PN-1 being found in high concentrations. In addition, colligin is an inducible serpin in the brain (Xi et al. 2000).

$\mathrm{PN}-1$, also known as glia-derived nexin, is localized around blood vessels and may act as the main thrombin inhibitor in the brain (Guenther et al. 1985; Stone et al. 1987). Despite its name, there is evidence that PN-1 expression is not limited to astrocytes as PN-1 mRNA has also been detected in neurons using in situ hybridization. PN-1 can modulate the mitogenic effects of thrombin and promote stellation of astrocytes (Cavanaugh et al. 1990). Delayed PN-1 up-regulation in the brain has been observed after cerebral ischemia and peripheral nerve lesion. PAI-1 is an inhibitor of urokinase (uPA) and tPA, but it can also inhibit thrombin (Mutch et al. 2001). PAI-1 may also inhibit thrombin production by inhibiting the cleavage of prothrombin to thrombin by activated factor X. Compared with PN-1, PAI-1 is a poor inhibitor of thrombin (second order rate constant $0.01 \times 10^{5} \mathrm{M}^{-1} \mathrm{~s}^{-1}$ vs. $15 \times 10^{5} \mathrm{M}^{-1} \mathrm{~s}^{-1}$ for PN-1). However, as with PN-1 and AT III, addition of a cofactor increases its effectiveness (second order rate constant $2.2 \times 10^{5} \mathrm{M}^{-1} \mathrm{~s}^{-1}$ in the presence of vitronectin) (Stone et al. 1987; Preissner et al. 1996).

Colligin, a collagen-binding serpin, is also called heat shock protein 47. Colligin localizes in the lumen of the endoplasmic reticulum and is induced in microglia and astrocytes after cerebral ischemia, subarachnoid hemorrhage and an intracerebral infusion of thrombin (Xi et al. 2000). The effects of colligin against thrombin may occur through restructuring the extracellular matrix rather than direct protease inhibition. Thrombomodulin (TM), an important thrombin-binding protein, is detected in endothelial cells and astrocytes (Pindon et al. 2000). TM inhibits thrombin by forming a TM-thrombin complex that then induces the activation of protein $\mathrm{C}$, an anticoagulatant. TM gene expression is increased in astrocytes after injury and this up-regulation might be mediated by thrombin via PAR-1 (Pindon et al. 2000). Sarker et al. reported that either a recombinant $\mathrm{TM}$ or a minimum functional domain of TM reduces thrombin-induced neuronal cell death (Sarker et al. 1999).

\section{Thrombin-induced brain tolerance}

Low concentrations of thrombin are neuroprotective in vitro and in vivo. In vitro studies have shown that thrombin protects rat primary astrocytes from hypoglycemia or oxidative stress induced cell death. Thrombin also protects rat primary hippocampal neurons from cell death produced by hypoglycemia, hypoxia or growth supplement deprivation (Vaughan et al. 1995; Striggow et al. 2000). In addition, thrombin attenuates neuronal cell death and modulates astrocyte reactivity induced by $\beta$-amyloid in vitro. The effect of thrombin on both neurons and astrocytes is mimicked by thrombin receptor-activating peptide and inhibited by two potent thrombin inhibitors, hirudin and protease nexin-1 (Pike et al. 1996). Furthermore, thrombin pretreatment prevents cell damage induced by a large dose of thrombin in vitro (Jiang et al. 2002a).

In vivo studies have shown that intracerebral infusion of a low dose of thrombin (thrombin preconditioning; TPC) reduces the brain injury that follows a subsequent intracerebral infusion of a high dose of thrombin, an intracerebral hemorrhage or cerebral ischemia (Xi et al. 1999; Masada et al. 2000; Xi et al. 2000). Thrombin pretreatment significantly attenuated the brain edema which normally follows the infusion of a large dose of thrombin. This effect was abolished by a thrombin inhibitor, hirudin (Xi et al. 1999). TPC may also be an important component of ischemic preconditioning as the thrombin inhibitor, hirudin, can block ischemic brain tolerance (Striggow et al. 2000).

Although the precise mechanisms of thrombin-induced brain tolerance to hemorrhagic and ischemic stroke are not known, activation of thrombin receptors, up-regulation of thrombin inhibitors and heat shock proteins in the brain may be associated with the induced tolerance (Xi et al. 1999; Xi et al. 2000; Hua et al. 2002; Jiang et al. 2002a).

The majority of cellular functions influenced by thrombin are mediated by thrombin receptors (PAR-1, -3 and -4 ) under normal conditions (Coughlin 2000). Thrombin receptor activation is involved in the protection induced by TPC as the effect is mimicked by a thrombin receptor agonist and suppressed by a thrombin receptor antagonist both in vivo and in vitro (Jiang et al. 2002a). Striggow et al. also found that a thrombin receptor-activating peptide mimicks the effects of a low concentration of thrombin (50 pM) in reducing ischemic neuronal injury in vitro (Striggow et al. 2000). Activation of thrombin receptor is associated with neuroprotection induced by thrombin (Jiang et al. 2002a). Prior activation of thrombin receptor by either thrombin receptor-activating peptide (TRAP) or by thrombin resulted in full protection of thrombin receptor mRNA levels under hypoxic conditions (Landau et al. 2000).

Endogenous thrombin inhibitors such as PAI-1 may be responsible for thrombin-induced brain protection. PAI-1 content is increased after intracerebral infusion of thrombin 
in a dose-dependent manner, as well as after intracerebral hemorrhage and cerebral ischemia. Hirudin blocked the up-regulation of PAI-1 after ICH indicating a role for thrombin (Ahn et al. 1999; Hua et al. 2002). PAI-1 appears to be neuroprotective as it reduces NMDA-induced neurotoxicity (Buisson et al. 1998) and ischemic infarct volume is increased in PAI-1 knockout mice (Nagai et al. 1999). However, the protective effect conferred by PAI-1 may not be a result of its action against thrombin alone because PAI-1 is also an inhibitor of tPA.

Induction of heat shock proteins (HSPs) may also contribute to thrombin-induced brain tolerance. HSP27 and HSP47 are both up-regulated after thrombin treatment (Xi et al. 1999, 2000). In vivo, TPC-induced brain tolerance against thrombin-induced brain edema appears to peak about seven days after thrombin treatment. This time delay for tolerance induction suggests that new protein synthesis is required. The timecourse of HSP27 (western blot) also found peak levels about seven days after TPC. The close temporal relationship between HSP27 expression and thrombininduced tolerance suggests that there may be a causal relationship. HSP27 can modulate the cytoskeleton by altering actin dynamics. It has been suggested that HSP27 protects against heat shock and oxidative stress through stabilization of actin filaments. Reorganization of the actin cytoskeleton may alter cell swelling following brain injuries (Xi et al. 1999). In addition, HSP27 may have other protective effects, including acting as a molecular chaperon and increasing levels of the antioxidant glutathione (Sharp et al. 1999).

The mechanisms by which HSP47 (colligin) up-regulation might be involved in attenuating ICH-induced brain edema are unclear. Colligin can inhibit thrombin but it is found in the endoplasmic reticulum. Instead, the effects of colligin against thrombin-induced injury may involve restructuring of the extracellular matrix rather than direct protease inhibition. Colligin is an essential factor for procollagen formation in the endoplasmic reticulum and prevents abnormal procollagen secretion. Procollagen is secreted to the extracellular space and forms collagen, a primary component of extracellular matrix. The extracellular matrix has been suggested as an important factor that regulates thrombin toxicity. In vitro, cells attached to an extracellular matrix are much less susceptible to thrombin-induced death (Cunningham and Donovan 1997).

\section{Thrombin-induced brain injury}

Although low concentrations of thrombin protect the brain from various kinds of injury, high concentrations cause brain damage. Thus, evidence indicates that thrombin plays an important role in inducing brain injury after intracerebral hemorrhage (Lee et al. 1996b; Xi et al. 1998). The brain edema induced by intracerebral infusion of $100 \mu \mathrm{L}$ of blood in the rat is roughly equivalent to that induced by the infusion of $5 \mathrm{U}$ of thrombin. Considering that the prothrombin in $100 \mu \mathrm{L}$ of blood could generate about $30 \mathrm{U}$ of thrombin, it appears that there is sufficient thrombin in a hematoma to induce brain injury. In addition, hirudin and $N$ - $\alpha$-(2-naphthalenesulfonylglycyl)-4-amidino-DL-phenylalaninepiperidide ( $\alpha$-NAPAP), two thrombin inhibitors, both inhibit edema formation in a rat intracerebral hemorrhage model (Lee et al. 1996a,b; Striggow et al. 2000).

There is evidence that thrombin also mediates brain injury in human $\mathrm{ICH}$, with perihematomal brain edema being less after systemic treatment with argatroban, a thrombin inhibitor (Hamada et al. 2000). Drug administration was only started $24 \mathrm{~h}$ after the hemorrhage to prevent rebleeding. Thus, although thrombin is produced immediately as blood clots following $\mathrm{ICH}$, some thrombin appears to be still present for at least $24 \mathrm{~h}$. Some of thrombin produced during coagulation may remain within the hematoma where it is associated with fibrin, and may be released slowly into the surrounding brain (Mutch et al. 2001). Alternately, prothrombin from plasma may enter the brain as a result of BBB disruption and be converted into thrombin. Thrombin may also contribute to brain injury in subarachnoid hemorrhage (SAH). Thrombin activity is increased in cerebrospinal fluid after $\mathrm{SAH}$ and is correlated with the degree of SAH and the development of vasospasm (Kasuya et al. 1998).

There is growing direct evidence suggesting that thrombin may also play crucial roles in brain injury after cerebral ischemia. Thrombin activity is increased in the ischemic core zone and prothrombin gene expression is also up-regulated several hours after middle cerebral artery occlusion in the rat (Xi et al. 2002). Intraventricular infusion of hirudin increases the survival of hippocampal CA1 neurons after global cerebral ischemia in gerbils (Striggow et al. 2000). Thrombin receptors are markedly up-regulated in hippocampus after oxygen/glucose deprivation in vitro (Striggow et al. 2001).

Systemic administration of thrombin inhibitors has been shown effective in experimental cerebral ischemia. Argatroban, a specific thrombin inhibitor, reduces infarction volume, improves neurological outcome and extends the therapeutic window for tPA treatment in a rat embolic model (Morris et al. 2001). In addition, argatroban reduces neurodegeneration and brain edema following bilateral common carotid artery occlusion with reperfusion in gerbils (Ohyama et al. 2001). Two other thrombin inhibitors, melagatran and inogatran, reduce the cortical infarction induced by photochemical reaction in rats (Mikulski et al. 2001). The extent to which ischemic protection with systemic thrombin inhibitors reflects vascular or parenchymal effects needs further study. Similarly, although there is debate on whether there is an increased risk for stroke in patients with the prothrombin G20210A gene mutation (which results in increased concentrations of plasma prothrombin; Wu and Tsongalis 2001), the question of whether this mutation might increase the 
severity of a stroke by increasing brain thrombin concentrations has not been examined.

High concentrations of thrombin are required to kill neurons and astrocytes in vitro. Thus, for example, $5 \mathrm{U} / \mathrm{mL}$ induces cell death in mixed neuronal/astrocyte cultures (Jiang et al. 2002a). It should be noted, however, that much lower concentrations of thrombin may cause cell death in stressed cells. Weinstein et al. examining Ad12 HER 10 cells, found that thrombin concentrations as low as $1.2 \times 10^{-3} \mathrm{U} / \mathrm{mL}$ caused cell death in hypoglycemic $(0.1 \mathrm{~mm}$ glucose) but not in normoglycemic conditions (Weinstein et al. 1998). This suggests that thrombin might cause brain injury even at very low levels during cerebral ischemia. Indeed, small doses of exogenous thrombin exacerbate ischemic brain injury in vivo (Xi et al. 2002).

Thrombin may cause brain injury by enhancing excitotoxicity, activating gelatinases and stimulating cytokine release. Glutamate-induced excitotoxicity is an important mechanism in ischemic brain injury, at least in animals. For example, MK 801, an NMDA antagonist, reduces infarct size after focal cerebral ischemia in the rat. Activation of PAR-1 potentiates NMDA receptor responses in CA1 pyramidal cells and the effect of thrombin can be mimicked by TRAP. Thrombin-induced NMDA receptor potentiation is reduced in PAR-1 knockout mice (Gingrich et al. 2000).

Thrombin activates gelatinase A (also called matrix metalloproteinases-2, MMP-2) in endothelial cells (Nguyen et al. 1999). MMPs are members of a family of zincdependent proteases that can degrade extracellular matrix and cause BBB disruption.

\section{Thrombin signaling pathways in brain injury and induced brain tolerance}

The fact that thrombin can reduce brain injury at low concentrations and enhance it at high concentrations raises the question of whether these effects are mediated by either the activation of different pathways or the differential stimulation of the same pathway? For example, protection and damage might result from stimulation of receptor and non-receptor mediated pathways or activation of different receptors. Alternately, whether thrombin causes brain injury or brain protection may depend on the extent to which one type of receptor is activated. Although this question has not been definitively answered, there is evidence that thrombininduced brain tolerance and thrombin-mediated brain damage may share the same initial signaling pathway involving activation of thrombin receptors (Donovan and Cunningham 1998).

Prior studies have implicated several different signaling cascades induced by thrombin receptor activation including activation of Ras and Rho, stimulation of MAPKs phosphorylation and release of intracellular calcium (Grand et al. 1996; Ubl and Reiser 1997; Dery et al. 1998; Donovan and
Cunningham 1998; Xi et al. 2001). Thrombin is able to activate Ras (an 189 amino acid peptide) and increases RhoA (a small GTP-binding protein) activity (Dery et al. 1998; Donovan and Cunningham 1998). RhoA activity increases in neurons and astrocytes in vitro after thrombin stimulation and exoenzyme C3 (RhoA inhibitor) blocks thrombininduced cell death and thrombin-induced protection against hypoglycemia (Donovan et al. 1997; Donovan and Cunningham 1998).

MAPKs are well-known cytoplasmatic signal transducers with an important role in thrombin-induced brain tolerance. In vivo, p44/42 MAPKs are activated in the brain after intracerebral infusion of thrombin. PD98059, a specific p44/ 42 MAPKs kinase inhibitor, abolished thrombin-induced activation of p44/42 MAPKs and also blocked thrombininduced brain tolerance (Xi et al. 2001). In vitro, thrombin treatment also activated p44/42 MAPKs and PD98059 completely blocked the cytoprotective effect of thrombin pretreatment, indicating that the p44/42 MAPK system mediates the neuroprotective effect induced by thrombin (Jiang et al. 2002a).

Calcium mobilization into the cell is related to thrombin signaling. Thrombin stimulates $\mathrm{IP}_{3} . \mathrm{IP}_{3}$ then regulates intracellular calcium content (Dery et al. 1998). Both thrombin and thrombin receptor activating peptide can stimulate calcium signaling in astrocytes (Ubl et al. 2000). In cultured hippocampal slices, Striggow et al. found that $50 \mathrm{pm}$ thrombin induces repetitive intracellular calcium spikes and is neuroprotective against oxygen and glucose deprivation. In contrast, $500 \mathrm{pm}$ thrombin causes a delayed single calcium spike and is not neuroprotective (Striggow et al. 2000).

Recently, we and others have found that hypoxia inducible factor- $1 \alpha(\mathrm{HIF}-1 \alpha)$ may be regulated by thrombin. Intracerebral infusion of thrombin up-regulates HIF- $1 \alpha$ protein levels in neurons. This effect appears to be specific, as it was blocked by a co-infusion of hirudin. HIF-1 $\alpha$ protein levels increased without changing HIF- $1 \alpha$ messenger RNA levels, indicating that thrombin up-regulates HIF- $1 \alpha$ by reducing HIF-1 $\alpha$ degradation (Jiang et al. 2002b). The thrombin effect is not likely to be mediated by hypoxia as we have previously found no reduction in cerebral blood flow after thrombin injections into the basal ganglia (Lee et al. 1997b). Other studies also found that HIF- $1 \alpha$ levels are up-regulated in vascular smooth muscle cells by thrombin (Richard et al. 2000). In addition, the experiments of Richard et al. indicate that HIF- $1 \alpha$ can be phosphorylated by p $44 / 42$ MAPKs and thrombin induces HIF-1 $\alpha$ up-regulation without a change of oxygen level (Richard et al. 2000).

HIF- $1 \alpha$ regulates the expression of a number of genes, including PAI-1 and heme oxygenase-1 (HO-1). The serpin PAI-1 is up-regulated by thrombin and may be neuroprotective, perhaps via its ability to inhibit thrombin and/or other serine proteases (Hua et al. 2002). HO-1, also known as 
HSP32, degrades heme into iron, carbon monoxide and biliverdin. Studies suggest that HO-1 is involved in cytoprotection from different kinds of injuries, although HO-1 up-regulation may induce brain injury by increasing cellular iron content (Sharp et al. 1999). In common with thrombin, HIF-1 $\alpha$ and HO-1 have implicated in both reducing and enhancing brain injury. The question of whether there is some adaptive advantage to the latter (e.g. the removal of dysfunctional cells) in some forms of injury, has yet to be answered.

Thus, in summary, thrombin acting on thrombin receptors at low concentration induces neuroprotection and at high concentration causes brain injury. Multi-signal transduction pathways are involved. Modulating thrombin activity and its inhibitors in the brain may establish novel therapeutic strategies for ischemic, hemorrhagic and traumatic brain injury. However, because of the dichotomy in the effects of thrombin on brain injury, it is essential to delineate the pathways involved in both the deleterious and the beneficial effects of thrombin on brain injury. Selective inhibition of the former is likely to prove a more effective therapeutic strategy than a direct thrombin inhibitor. The current availability of PAR-1, -3 and -4 knockout mice should greatly facilitate this process.

In addition, there is still the largely unanswered question as to what is the normal function of prothrombin, thrombin and thrombin receptors in the brain. Is there a role limited to situations of brain injury? Or, as has been proposed for tPA (Seeds et al. 1995), do they have a role in normal brain function such as brain plasticity? Evidence on their effects on astrocyte and neuron morphology and ion channel activity (Grand et al. 1996), suggests that this may be the case.

\section{Acknowledgements}

GX and RK were supported in this study by grants NS-39866, NS-34709 and NS-17760 from the National Institutes of Health and Grant-In-Aid 0255721N from the American Heart Association. GR was supported by grants from the Deutsche Forschungsgemeinschaft (Re563/11-1 and Re847/3-1) and Land Sachsen-Anhalt 2923 A.

\section{References}

Ahn M. Y., Zhang Z. G., Tsang W. and Chopp M. (1999) Endogenous plasminogen activator expression after embolic focal cerebral ischemia in mice. Brain Res. 837, 169-176.

Buisson A., Nicole O., Docagne F., Sartelet H., Mackenzie E. T. and Vivien D. (1998) Up-regulation of a serine protease inhibitor in astrocytes mediates the neuroprotective activity of transforming growth factor beta1. FASEB J. 12, 1683-1691.

Cavanaugh K. P., Gurwitz D., Cunningham D. D. and Bradshaw R. A. (1990) Reciprocal modulation of astrocyte stellation by thrombin and protease nexin-1. J. Neurochem. 54, 1735-1743.

Citron B. A., Smirnova I. V., Arnold P. M. and Festoff B. W. (2000) Upregulation of neurotoxic serine proteases, prothrombin, and protease-activated receptor 1 early after spinal cord injury. J. Neurotrauma 17, 1191-1203.

Coughlin S. R. (2000) Thrombin signalling and protease-activated receptors. Nature 407, 258-264.

Cunningham D. D. and Donovan F. M. (1997) Regulation of neurons and astrocytes by thrombin and protease nexin-1. Relationship to brain injury. Adv. Exp Med. Biol. 425, 67-75.

Dery O., Corvera C. U., Steinhoff M. and Bunnett N. W. (1998) Proteinase-activated receptors: novel mechanisms of signaling by serine proteases. Am. J. Physiol. 274, C1429-C1452.

Dihanich M., Kaser M., Reinhard E., Cunningham D. and Monard D. (1991) Prothrombin mRNA is expressed by cells of the nervous system. Neuron 6, 575-581.

Donovan F. M. and Cunningham D. D. (1998) Signaling pathways involved in thrombin-induced cell protection. J. Biol. Chem 273, 12746-12752.

Donovan F. M., Pike C. J., Cotman C. W. and Cunningham D. D. (1997) Thrombin induces apoptosis in cultured neurons and astrocytes via a pathway requiring tyrosine kinase and RhoA activities. J. Neurosci. 17, 5316-5326.

Figueroa B. E., Keep R. F., Betz A. L. and Hoff J. T. (1998) Plasminogen activators potentiate thrombin-induced brain injury. Stroke 29, $1202-1207$.

Gingrich M. B. and Traynelis S. F. (2000) Serine proteases and brain damage - is there a link?. Trends Neurosciences 23, 399-407.

Gingrich M. B., Junge C. E., Lyuboslavsky P. and Traynelis S. F. (2000) Potentiation of NMDA receptor function by the serine protease thrombin. J. Neurosci. 20, 4582-4595.

Grand R. J., Turnell A. S. and Grabham P. W. (1996) Cellular consequences of thrombin-receptor activation. Biochem. J. 313, 353-368.

Guenther J., Nick H. and Monard D. (1985) A glia-derived neuritepromoting factor with protease inhibitory activity. $E M B O \mathrm{~J} . \mathbf{4}$, 1963-1966.

Hamada R., Matsuoka H., Wagner K. R., Broderick J. P., deCourtenMyers G. M., Xi G., Keep R. F. and Hoff J. T. (2000) Antithrombin therapy for intracerebral hemorrhage. Stroke 31, 794-795.

Hua Y., Xi G., Keep R. F., Wu J., Jiang Y. and Hoff J. T. (2002) Plasminogen activator inhibitor-1 induction after experimental intracerebral hemorrhage. J. Cereb. Blood Flow Metab. 22, 55-61.

Jiang Y., Wu J., Hua Y., Keep R. F., Xiang J., Hoff J. T. and Xi G. (2002a) Thrombin-receptor activation and thrombin-induced brain tolerance. J. Cereb. Blood Flow Metab. 22, 404-410.

Jiang Y., Wu J., Keep R. F., Hua Y., Hoff J. T. and Xi G. (2002b) Hypoxia-inducible factor-1a accumulation in the brain after experimental intracerebral hemorrhage. J. Cereb. Blood Flow Metab. 22, 689-696.

Kasuya H., Shimizu T. and Takakura K. (1998) Thrombin activity in CSF after SAH is correlated with the degree of SAH the persistence of subarachnoid clot and the development of vasospasm. Acta Neurochir. 140, 579-584.

Landau E., Tirosh R., Pinson A., Banai S., Even-Ram S., Maoz M., Katzav S. and Bar-Shavit R. (2000) Protection of thrombin receptor expression under hypoxia. J. Biol. Chem. 275, 2281-2287.

Lee K. R., Betz A. L., Kim S., Keep R. F. and Hoff J. T. (1996a) The role of the coagulation cascade in brain edema formation after intracerebral hemorrhage. Acta Neurochir. 138, 396-400.

Lee K. R., Colon G. P., Betz A. L., Keep R. F., Kim S. and Hoff J. T. (1996b) Edema from intracerebral hemorrhage: the role of thrombin. J. Neurosurg. 84, 91-96.

Lee K. R., Drury I., Vitarbo E. and Hoff J. T. (1997a) Seizures induced by intracerebral injection of thrombin: a model of intracerebral hemorrhage. J. Neurosurg. 87, 73-78.

Lee K. R., Kawai N., Kim S., Sagher O. and Hoff J. T. (1997b) Mechanisms of edema formation after intracerebral hemorrhage: 
effects of thrombin on cerebral blood flow, blood-brain barrier permeability, and cell survival in a rat model. J. Neurosurg. 86, $272-278$.

Masada T., Xi G., Hua Y. and Keep R. F. (2000) The effects of thrombin preconditioning on focal cerebral ischemia in rats. Brain Res. 867, 173-179.

Mikulski A., Elg M. and Gustafsson D. (2001) The effects of oral and intravenous direct thrombin inhibitors on the size of photochemically induced cortical infarction in rats. Thromb. Res. 101, 477-482.

Moller T., Hanisch U.K. and Ransom B. R. (2000) Thrombin-induced activation of cultured rodent microglia. J. Neurochem. 75, 15391547.

Morris D. C., Zhang L., Zhang Z. G., Lu M., Berens K. L., Brown P. M. and Chopp M. (2001) Extension of the therapeutic window for recombinant tissue plasminogen activator with argatroban in a rat model of embolic stroke. Stroke 32, 2635-2640.

Mutch N. J., Robbie L. A. and Booth N. A. (2001) Human thrombi contain an abundance of active thrombin. Thromb. Haemostasis 86, 1028-1034.

Nagai N., De Mol M., Lijnen H. R., Carmeliet P. and Collen D. (1999) Role of plasminogen system components in focal cerebral ischemic infarction: a gene targeting and gene transfer study in mice. Circulation 99, 2440-2444.

Nguyen M., Arkell J. and Jackson C. J. (1999) Thrombin rapidly and efficiently activates gelatinase $\mathrm{A}$ in human microvascular endothelial cells via a mechanism independent of active MT1 matrix metalloproteinase. Lab. Invest. 79, 467-475.

Nishino A., Suzuki M., Ohtani H., Motohashi O., Umezawa K., Nagura H. and Yoshimoto T. (1993) Thrombin may contribute to the pathophysiology of central nervous system injury. J. Neurotrauma 10, 167-179.

Ohyama H., Hosomi N., Takahashi T., Mizushige K. and Kohno M. (2001) Thrombin inhibition attenuates neurodegeneration and cerebral edema formation following transient forebrain ischemia. Brain Res. 902, 264-271.

Pike C. J., Vaughan P. J., Cunningham D. D. and Cotman C. W. (1996) Thrombin attenuates neuronal cell death and modulates astrocyte reactivity induced by beta-amyloid in vitro. J. Neurochem. 66, 1374-1382.

Pindon A., Berry M. and Hantai D. (2000) Thrombomodulin as a new marker of lesion-induced astrogliosis: involvement of thrombin through the G-protein-coupled protease-activated receptor-1. J. Neurosci. 20, 2543-2550.

Preissner K. T., de Boer H., Pannekoek H. and de Groot P. G. (1996) Thrombin regulation by physiological inhibitors: the role of vitronectin. Seminars Thromb. Hemostasis 22, 165-172.

Richard D. E., Berra E. and Pouyssegur J. (2000) Nonhypoxic pathway mediates the induction of hypoxia-inducible factor 1alpha in vascular smooth muscle cells. J. Biol. Chem. 275, 26765-26771.

Riek-Burchardt F., Striggow F., Henrich-Noack P., Reiser G. and Reymann K. G. (2002) Increase of prothrombin-mRNA after global ischemia in rats, with constant expression of protease nexin1 and protease-activated receptors. Neurosci. Lett. 329, 181-184.

Sarker K. P., Abeyama K., Nishi J., Nakata M., Tokioka T., Nakajima T., Kitajima I. and Maruyama I. (1999) Inhibition of thrombin-induced neuronal cell death by recombinant thrombomodulin and E5510, a synthetic thrombin receptor signaling inhibitor. Thromb. Haemostasis 82, 1071-1077.

Seeds N. W., Williams B. L. and Bickford P. C. (1995) Tissue plasminogen activator induction in Purkinje neurons after cerebellar motor learning. Science 270, 1992-1994.

Sharp F. R., Massa S. M. and Swanson R. A. (1999) Heat-shock protein protection. TINS 22, 97-99.
Shikamoto Y. and Morita T. (1999) Expression of factor X in both the rat brain and cells of the central nervous system. FEBS Lett. 463, 387389.

Stone S. R., Nick H., Hofsteenge J. and Monard D. (1987) Glial-derived neurite-promoting factor is a slow-binding inhibitor of trypsin, thrombin, and urokinase. Arch. Biochem. Biophys. 252, 237-244.

Striggow F., Riek M., Breder J., Henrich-Noack P., Reymann K. G. and Reiser G. (2000) The protease thrombin is an endogenous mediator of hippocampal neuroprotection against ischemia at low concentrations but causes degeneration at high concentrations. Proc. Natl Acad. Sci. USA 97, 2264-2269.

Striggow F., Riek-Burchardt M., Kiesel A., Schmidt W., Henrich-Noack P., Breder J., Krug M., Reymann K. G. and Reiser G. (2001) Four different types of protease-activated receptors are widely expressed in the brain and are up-regulated in hippocampus by severe ischemia. Eur. J. Neurosci. 14, 595-608.

The NINDS rt-PA stroke study Group (1995) Tissue plasminogen activator for acute ischemic stroke. The National Institute of Neurological Disorders and Stroke rt-PA Stroke Study Group. New Eng. J. Med. 333, 1581-1587.

Ubl J. J. and Reiser G. (1997) Characteristics of thrombin-induced calcium signals in rat astrocytes. Glia 21, 361-369.

Ubl J. J., Sergeeva M. and Reiser G. (2000) Desensitisation of proteaseactivated receptor-1 (PAR-1) in rat astrocytes: evidence for a novel mechanism for terminating $\mathrm{Ca} 2+$ signalling evoked by the tethered ligand. J. Physiol. 2, 319-330.

Vaughan P. J., Pike C. J., Cotman C. W. and Cunningham D. D. (1995) Thrombin receptor activation protects neurons and astrocytes from cell death produced by environmental insults. J. Neurosci. 15, 5389-5401.

Vu T. K., Hung D. T., Wheaton V. I. and Coughlin S. R. (1991) Molecular cloning of a functional thrombin receptor reveals a novel proteolytic mechanism of receptor activation. Cell 64, $1057-1068$.

Wang H., Ubl J. J. and Reiser G. (2002) Four subtypes of proteaseactivated receptors, co-expressed in rat astrocytes, evoke different physiological signaling. Glia 37, 53-63.

Weinstein J. R., Gold S. J., Cunningham D. D. and Gall C. M. (1995) Cellular localization of thrombin receptor mRNA in rat brain: expression by mesencephalic dopaminergic neurons and codistribution with prothrombin mRNA. J. Neurosci. 15, 29062919.

Weinstein J. R., Lau A. L., Brass L. F. and Cunningham D. D. (1998) Injury-related factors and conditions down-regulate the thrombin receptor (PAR-1) in a human neuronal cell line. J. Neurochem. 71, 1034-1050.

Wu A. H. and Tsongalis G. J. (2001) Correlation of polymorphisms to coagulation and biochemical risk factors for cardiovascular diseases. Am. J. Cardiol. 87, 1361-1366.

Xi G., Wagner K. R., Keep R. F., Hua Y., de Courten-Myers G. M., Broderick J. P., Brott T. G. and Hoff J. T. (1998) The role of blood clot formation on early edema development following experimental intracerebral hemorrhage. Stroke 29, 2580-2586.

Xi G., Keep R. F., Hua Y., Xiang J. M. and Hoff J. T. (1999) Attenuation of thrombin-induced brain edema by cerebral thrombin preconditioning. Stroke 30, 1247-1255.

Xi G., Hua Y., Keep R. F. and Hoff J. T. (2000) Induction of colligin may attenuate brain edema following intracerebral hemorrhage. Acta Neurochir. 76, 501-505.

Xi G., Hua Y., Keep R. F., Duong H. K. and Hoff J. T. (2001) Activation of p44/42 mitogen activated protein kinases in thrombin-induced brain tolerance. Brain Res. 895, 153-159.

Xi G., Hua Y., Wu J. and Keep R. F. (2002) Increase of brain thrombin concentration in cerebral ischemia. Stroke 33, 399. 